\title{
Regression of Multiple Meningiomas after Discontinuation of Chronic Hormone Therapy: A Case Report
}

\author{
Maryam N. Shahin ${ }^{1}$ Stephen G. Bowden ${ }^{1} \quad$ Nasser K. Yaghi ${ }^{1} \quad$ Jacob H. Bagley ${ }^{1}$ Seunggu J. Han ${ }^{1}$ \\ Elena V. Varlamov ${ }^{1,2}$ Marjorie R. Grafe ${ }^{3}$ Justin S. Cetas ${ }^{1,4}$
}

${ }^{1}$ Department of Neurological Surgery, Oregon Health \& Science University, Portland, Oregon, United States

2 Division of Endocrinology, Diabetes and Clinical Nutrition, Department of Medicine (Endocrinology), Oregon Health \& Science University, Portland, Oregon, United States

${ }^{3}$ Department of Pathology, Oregon Health \& Science University, Portland, Oregon, United States

${ }^{4}$ Operative Care Division, Portland Veterans Affairs Medical Center, Portland, Oregon, United States

J Neurol Surg Rep 2021;82:e38-e42.
Address for correspondence Justin S. Cetas, MD, PhD, Department of Neurological Surgery, Oregon Health \& Science University, 3303 South Bond Avenue, Mail Code CH8N, Portland, OR 97239, United States (e-mail: cetasj@ohsu.edu).

\begin{abstract}
Keywords

- meningioma

- estrogen

- progesterone

- endometriosis

Introduction Meningiomas are more common in females and frequently express progesterone and estrogen receptors. Recent studies have revealed a high incidence of meningiomas in situations in which estrogen/progesterone levels are increased such as pregnancy, gender reassignment therapy, and fertility treatment. While the relationship remains unclear and controversial, these findings suggest exposure to high levels of endogenous or exogenous hormones may increase the risk of developing a meningioma.

Patients and Methods A 40-year-old female with a history of endometriosis treated with chronic progesterone therapy presented with a visual deficit and was found to have multiple meningiomas, which regressed after cessation of exogenous progesterone.

Conclusion A history of chronic hormone therapy should be included when evaluating patients diagnosed with meningiomas, particularly at a younger age and with multiple meningiomas. Cessation of exogenous progesterone resulting in regression of meningiomas suggests a direct action of progesterone on growth. Future studies are warranted to better elucidate this relationship.
\end{abstract}

\section{Introduction}

Meningiomas are the most common primary central nervous system tumor and are known to express both estrogen and progesterone receptors (PRs). ${ }^{1}$ There is a female preponderance, with female-to-male ratio reported as high as 3.15:1 during reproductive age. ${ }^{2}$ Together, these characteristics led to the theory that sex hormones may play a role in the etiology and pathogenesis of meningiomas. While a correlation between meningioma and pregnancy is now well established, ${ }^{3-5}$ the association between meningioma and other sources of potential hormone exposure, such as gender received

February 21, 2020

accepted

October 28, 2020
DOI https://doi.org/ $10.1055 / \mathrm{s}-0041-1735553$ ISSN 2193-6358.

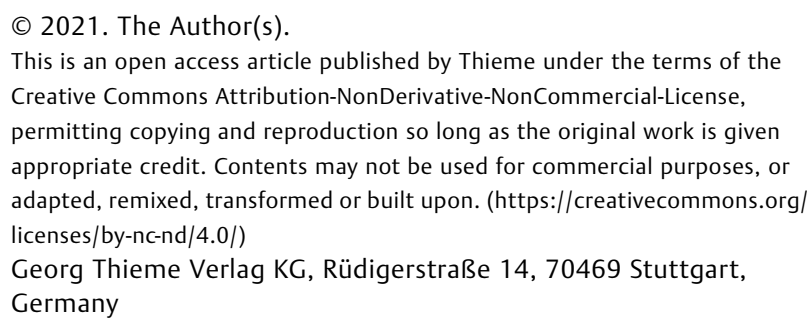


reassignment therapy or fertility treatment, has gained attention more recently. ${ }^{6-10}$ Endometriosis is a chronic disease characterized by endometrial tissue outside of the uterus, potentially requiring life-long treatment with hormone-based therapy. Treatment strategies include estrogens, progestins, androgens, gonadotropin-releasing hormone agonists, or antagonists and aromatase inhibitors. Progestin-based therapy aims to inhibit the growth of endometrial tissue by inducing an atrophic state. ${ }^{11}$ Here, we describe a case of a patient with endometriosis found to have multiple and symptomatic meningiomas after over 20 years of progesterone therapy and subsequent tumor volume shrinkage after cessation.

\section{Patients and Methods}

\section{Patient Information}

A 40-year-old female with a history of cervical cancer and endometriosis managed with at least 20 years of progesterone therapy (megestrol) presented with 4 to 5 months of progressively decreasing visual acuity in her right eye. She was evaluated by ophthalmology at an outside institution and found to have a temporal field deficit in her right eye, which prompted referral to our institution and recommen- dation for brain imaging. Of note, she described baseline poor acuity since childhood in her left eye corrected with glasses. The patient was consented per institution standard of care for rendering of medical services/surgical services/sedation; institutional review board approval is not required and was not sought.

\section{Imaging Study}

Magnetic resonance imaging (MRI) brain demonstrated multiple dural-based masses (at least five); the largest of which was along the planum sphenoidale with extension into the sella and displacement of the optic nerves (- Fig. 1).

\section{Medical History}

There was no history of previous radiation to the head/neck, thyroid disease, or syndromes such as neurofibromatosis-2 (NF-2). On physical exam, she did not have any cafe au lait spots or NF-2 related skin lesions aside from sparse skin tags. The patient denied ever been diagnosed with cataracts or retinal hamartomas. Her past medical history included ureterolithiasis, cholecystitis treated with cholecystectomy, and cervical cancer treated with cervical cone biopsy. There was no known family history of intracranial masses, including meningioma. The patient was a single nulligravida

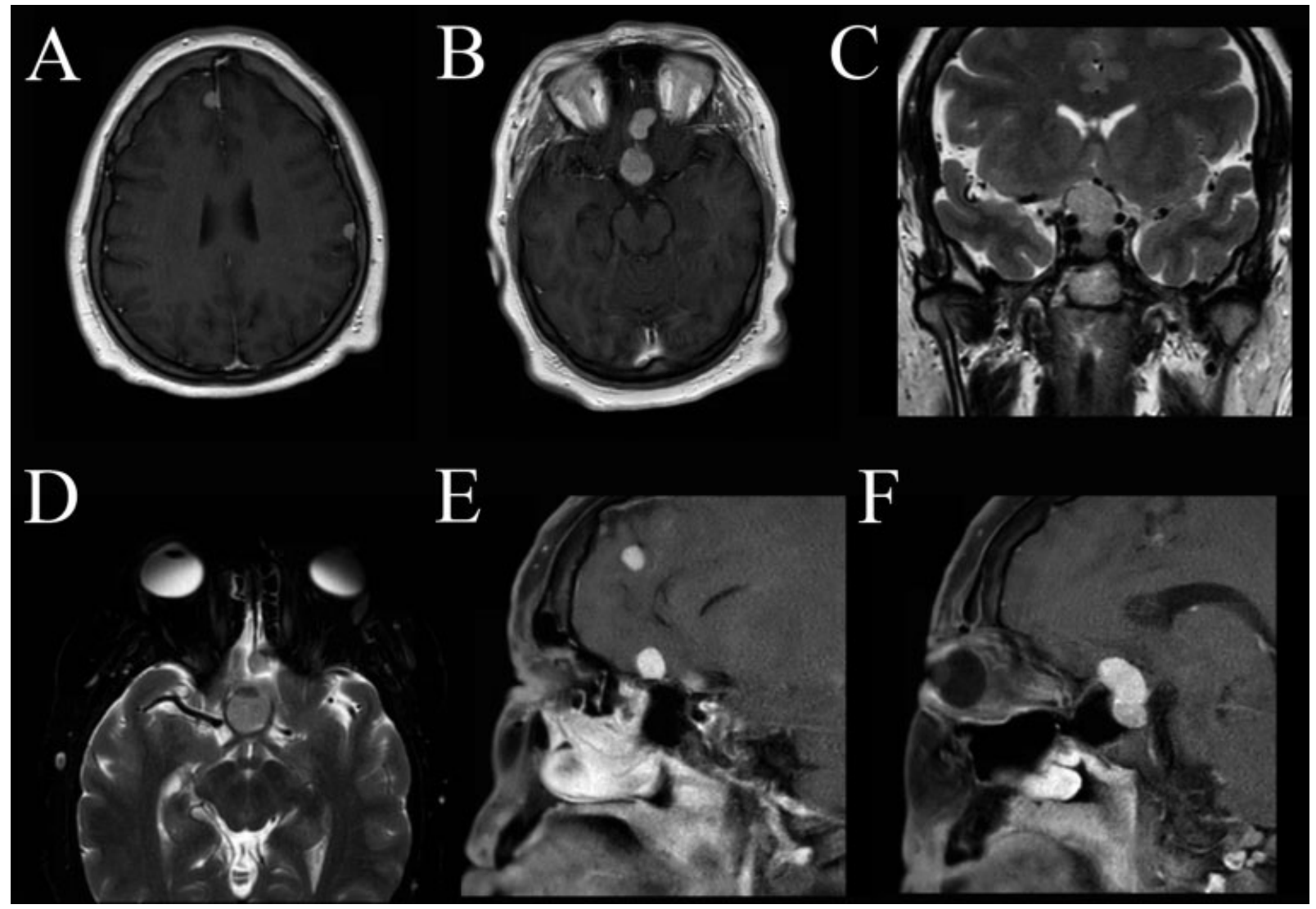

Fig. 1 Preoperative imaging. Two axial T1-weighted MRI slices (A, B) demonstrating multiple dural based masses at the convexity, parafalcine dura, and skull base. Coronal and axial T2-weighted MRI slices (C, D) demonstrating the symptomatic sellar lesion with obvious displacement of the prechiasmatic optic nerves, as well as mass effect and compression of the right greater than left optic chiasm. The dominant planum sphenoidale dural-based mass abuts the bilateral clinoid internal carotid arteries and right A1 segment of the anterior cerebral artery. Two sagittal T1-weighted MRI slices (E, F) depicting multiple lesions with the largest of which appearing to originate from the planum sphenoidale with extension into the sella. MRI, magnetic resonance imaging. 
female. She denied current or prior tobacco, alcohol, or drug use. Her neurologic exam was significant for an inability to count fingers in the temporal and superior nasal visual field using her right eye. She was able to count fingers in all visual fields using her left eye. Pupils were equal, round, and reactive to light with conjugate gaze (no nystagmus and no ptosis). The remainder of the neurological exam was unremarkable. Given the visual deficit, surgical resection was indicated.

\section{Surgical Course}

An expanded endoscopic endonasal approach was used to expose the tuberculum sellae and anterior cranial fossa. Using microdissection, a plane was established between the meningioma and infundibulum and the pituitary stalk remained intact and undisturbed. The meningioma was removed without complication. The dura was repaired using a dural allograft. She had an uncomplicated postoperative course and was discharged home on postoperative day 5 .

\section{Histopathological Analysis}

Histological examination revealed a World Health Organization (WHO) grade I meningothelial meningioma, which stained diffusely positive for PR and somatostatin 2 receptor (SSTR2A), with estrogen receptor (ER) positivity in a subset of cells (-Fig. 2). This is consistent with the majority of lower grade meningiomas expressing PR. However, the positivity for ER is rare and frequently associated with higher grade lesions. ${ }^{1}$

Immunohistochemical stains were performed on a Ventana Benchmark Ultra, using the following antibodies: SSTR2, 1:200, clone EP149 rabbit monoclonal, Cell Marque SigmaAldrich, St. Louis, MO; anti-ER, prediluted Ventana Medical Systems, clone SP1 rabbit monoclonal, Roche Tissue Diagnostics, Indianapolis, IN; anti-PR, prediluted Ventana

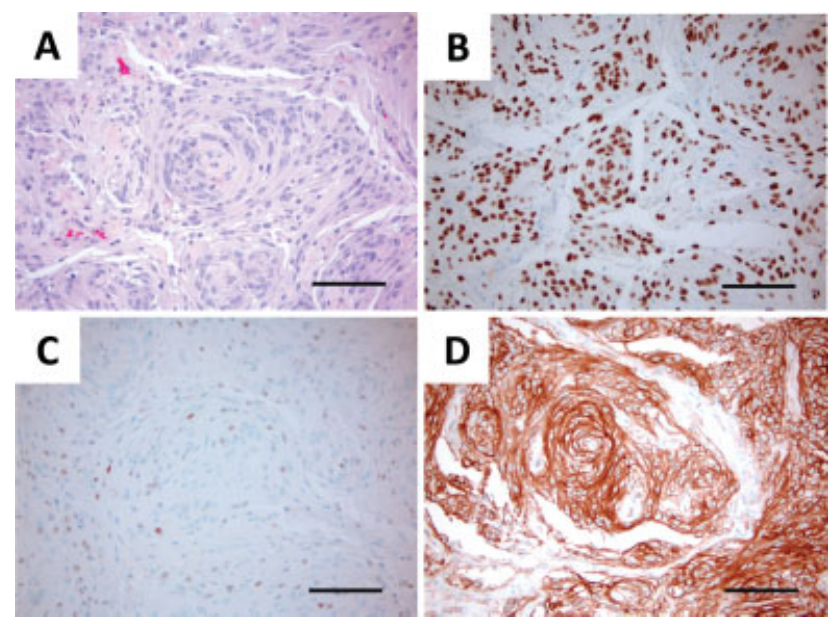

Fig. 2 Histological sections demonstrating (A) hematoxylin and eosin staining as well as immunohistochemistry for (B) progesterone receptor, $(C)$ estrogen receptor, and (D) somatostatin 2 a receptor at $\times 20$ from a patient with a history of chronic progesterone treatment for endometriosis and a World Health Organization grade 1 meningioma.
Medical Systems, clone 1E2 rabbit monoclonal, Roche Tissue Diagnostics, Indianapolis, IN.

\section{Postoperative Course}

The patient was seen at 3 months postoperatively. Visual fields were full to confrontation bilaterally. This was an improvement from preoperative temporal and superior nasal visual field deficit in the right eye. However, visual acuity bilaterally remained grossly poor, with the right eye worse than the left. A consult to ophthalmology for formal visual testing was placed at this time. Follow-up demonstrated debulking of the tuberculum sellae and olfactory groove lesions with decreased mass effect on the optic chiasm and pituitary gland with the infundibulum restored to midline.

\section{Follow-Up}

Megestrol was discontinued 5 months postoperatively after receiving clearance from her gynecologist. Because megestrol is known to suppress hypothalamic-pituitary-adrenal axis, $^{12}$ a $1 \mathrm{mcg}$ adrenocorticotrophin stimulation test was performed to assess the axis, and it confirmed secondary adrenal insufficiency. The patient was commenced on hydrocortisone replacement upon discontinuation of megestrol. Follow-up imaging 4 months after discontinuing megestrol, demonstrated interval decrease in size in multiple meningiomas, particularly the right anterior falcine, left parietal and left lateral frontal lesions (-Fig. 3).

\section{Discussion}

We present, to our knowledge, the first report of a meningioma in the setting of endometriosis managed with chronic progesterone therapy (megestrol) and subsequent regression upon exogenous hormone cessation. This patient was diagnosed with multiple meningiomas at 40 years of age, which is younger than the median age at diagnosis of meningioma of 55.9 years old. ${ }^{2}$ After discontinuing megestrol therapy, we found regression in tumor volume (-Fig. $\mathbf{3}$ ).

\section{Multiple Meningiomas}

Multiple meningiomas can occur sporadically or as part of a syndrome, such as NF-2. Multiple meningiomas represent 1 to $10 \%$ of patients diagnosed with meningiomas; however, the natural history of multiple meningiomas remains poorly understood and limited to small case series. ${ }^{13-15}$ The largest case series to date describes a cohort of 12 patients with multiple meningiomas, presenting at an average age at diagnosis of 37 years of age (range $=19-61$ years) ${ }^{15}$ However, in a similar series with 10 patients, the mean age at diagnosis was 50 years of age (range $=32-72$ years). ${ }^{14}$

\section{Endometriosis}

A relationship between endometriosis and meningioma was described by Claus et al, who compared personal and firstdegree family histories of 1,124 patients with meningioma. This cohort demonstrated an increased risk of meningioma in patients with a history of endometriosis over age-matched controls (odds ratio [OR]: $1.5,95 \%$ confidence interval $[\mathrm{CI}]$ : 


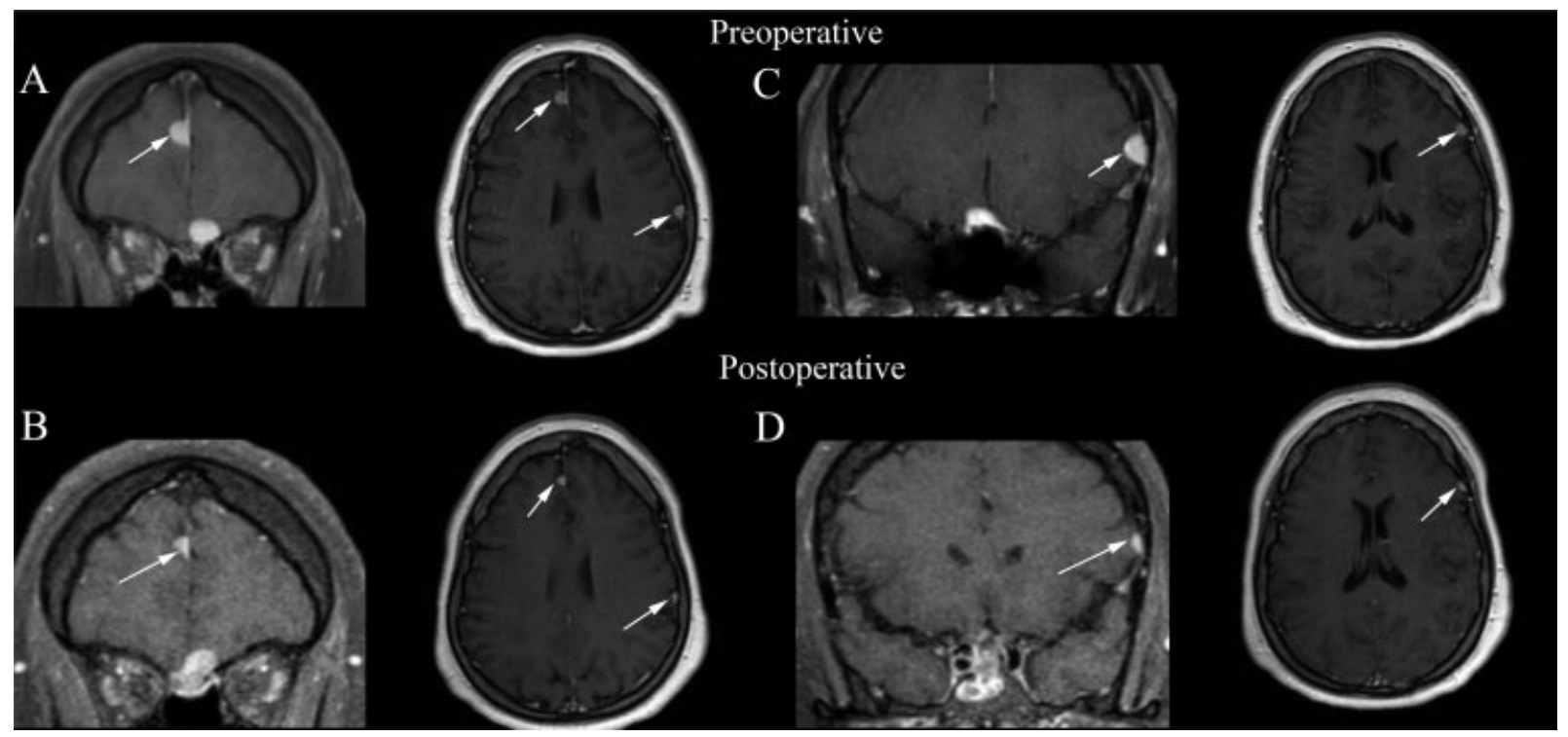

Fig. 3 Coronal and axial T1-weighted magnetic resonance imaging slices 10 months postoperative and 4 months postmegestrol cessation demonstrating interval decrease in size of multiple meningiomas, including: right anterior falx (A) $11 \mathrm{~mm}$, now (B) $5 \times 4 \times 8 \mathrm{~mm}$, left parietal calvarial (A) $7 \times 10 \mathrm{~mm}$, now (B) $3 \times 5 \mathrm{~mm}$ and lateral frontal calvarial based lesion (C) $8 \times 11 \mathrm{~mm}$, and now (D) $7 \times 4 \mathrm{~mm}$.

1.5-2.1). ${ }^{16}$ Women in this cohort were also more likely to report other hormonally related conditions such as uterine fibroid tumors (OR: $1.2,95 \% \mathrm{CI}: 1.0-1.5$ ) and breast cancer (OR: $1.3,95 \%$ CI: $0.8-2.3)$.

\section{Hormones and Meningioma}

Approximately, 70\% of meningiomas express PR and 30\% express ERs. Given the expression of estrogen and PRs, antihormonal therapy has been investigated for use in the treatment of meningioma. The competitive PR antagonist, mifepristone, inhibits meningioma cell growth in vitro 17,18 and has demonstrated a therapeutic effect in vivo in select patients with unresectable meningiomas. ${ }^{19}$ However, the only prospective study using long-term administration of antiprogestin for unresectable meningiomas failed to identify a therapeutic advantage between treatment groups. ${ }^{20}$

\section{Hormone Exposure}

Recently, several series have called attention to scenarios where increased hormonal levels, either endogenous or exogenous, correlate with meningioma growth and progression. Pregnancy drives an increase in both endogenous progesterone and estrogen levels. Several reports describe meningiomas rapidly growing and becoming symptomatic leading to diagnosis during pregnancy, with occasional regression of the tumor postpartum. ${ }^{3-5}$ The relationship between exogenous hormone exposure and meningioma has been studied in a myriad of settings, including oral contraception, fertility treatment, and gender reassignment. ${ }^{6-10,21}$ There has been one case report documenting shrinkage of multiple intracranial meningiomas after the cessation of long-term megestrol acetate therapy in the setting of breast carcinoma. ${ }^{22}$ Using progesterone-only contraception is associated with increased recurrence of WHO grade I meningiomas ( 33.3 vs. $19.6 \%$ ) as well as decreased time to recurrence (18 vs. 32 months). Peyre et al compared
40 female meningioma patients with a history of long-term progestin therapy to a control group of female meningioma patients, and similarly found significant differences in patient and tumor characteristics. Patients exposed to progestin therapy were younger at tumor onset (mean 48 vs. 58 years), and more often had multiple/multifocal meningiomas (48 vs. 5\%). Furthermore, meningiomas in the progestin therapy cohort had a shift in their mutational landscape, which may suggest progestin selects for specific molecular profiles. ${ }^{23}$ An institutional experience of history of fertility treatment in the setting of meningioma found patients with a history of any type of hormonal fertility treatments similarly presented at a younger mean age compared with those who had not (51.8 vs. 57.3 years), and on multivariate analysis were more likely to have multiple meningiomas (OR: 4.97, 95\% CI: 1.4-18.1) and convexity/falx meningiomas (OR: 4.45, 95\% CI: 1.7-11.5). ${ }^{24}$ Lastly, meningioma in the setting of gender reassignment therapy has been limited to case reports $8,21,25,26$ including a recent report of two maleto-female transsexual males on long-term hormone replacement therapy who both presented with visual disturbances leading to the discovery of multiple meningiomas, including parasellar meningiomas. ${ }^{25}$

\section{Conclusion}

The potential role of progesterone in meningioma pathogenesis should be taken into account when considering initiation of life-long progesterone therapy in a patient. While this patient's multiple meningiomas are not definitively connected to chronic progesterone use, her young age, finding of multiple meningiomas, and especially their subsequent regression upon cessation of exogenous hormone use do support this notion. ${ }^{23,24}$ Considering the relationship between hormone exposure and meningioma and the increased risk endometriosis confers, patients with 
endometriosis may benefit from screening prior to initiating hormone-based therapy and/or serial imaging throughout treatment. Further investigation is required to clearly understand this relationship and how to mitigate potential risks.

\section{Funding \\ None.}

\section{Conflict of Interest}

None declared.

\section{References}

1 Commins DL, Atkinson RD, Burnett ME. Review of meningioma histopathology. Neurosurg Focus 2007;23(04):E3

2 Wiemels J, Wrensch M, Claus EB. Epidemiology and etiology of meningioma. J Neurooncol 2010;99(03):307-314

3 Chacko JG, Miller JL, Angtuaco EJ. Spontaneous postpartum resolution of vision loss caused by a progesterone receptorpositive tuberculum sellae meningioma. J Neuroophthalmol 2010;30(02):132-134

4 Chakravarthy V, Kaplan B, Gospodarev V, Myers H, De Los Reyes K, Achiriloaie A. Houdini tumor: case report and literature review of pregnancy-associated meningioma. World Neurosurg 2018;114: e1261-e1265

5 Lusis EA, Scheithauer BW, Yachnis AT, et al. Meningiomas in pregnancy: a clinicopathologic study of 17 cases. Neurosurgery 2012;71(05):951-961

6 Claus EB, Black PM, Bondy ML, et al. Exogenous hormone use and meningioma risk: what do we tell our patients? Cancer 2007;110 (03):471-476

7 Claus EB, Calvocoressi L, Bondy ML, Wrensch M, Wiemels JL, Schildkraut JM. Exogenous hormone use, reproductive factors, and risk of intracranial meningioma in females. J Neurosurg 2013; 118(03):649-656

8 Deipolyi AR, Han SJ, Parsa AT. Development of a symptomatic intracranial meningioma in a male-to-female transsexual after initiation of hormone therapy. J Clin Neurosci 2010;17(10):1324-1326

9 Gazzeri R, Galarza M, Gazzeri G. Growth of a meningioma in a transsexual patient after estrogen-progestin therapy. $\mathrm{N}$ Engl J Med 2007;357(23):2411-2412

10 Harland TA, Freeman JL, Davern M, et al. Progesterone-only contraception is associated with a shorter progression-free survival in premenopausal women with WHO Grade I meningioma. J Neurooncol 2017;0(00):1-7

11 Practice T, Medicine RPractice Committee of the American Society for Reproductive Medicine. Treatment of pelvic pain associated with endometriosis: a committee opinion. Fertil Steril 2014;101 (04):927-935

12 Ron IG, Soyfer V, Goldray D, Inbar MJ, Weisman Y. A low-dose adrenocorticotropin test reveals impaired adrenal function in cancer patients receiving megestrol acetate therapy. Eur J Cancer 2002;38(11):1490-1494

13 Koech F, Orege J, Ndiangui F, Macharia B, Mbaruku N. Multiple intracranial meningiomas: a review of the literature and a case report. Case Rep Surg 2013;2013:131962

14 Sheehy JP, Crockard HA. Multiple meningiomas: a long-term review. J Neurosurg 1983;59(01):1-5

15 Wong RH, Wong AK, Vick N, Farhat HI. Natural history of multiple meningiomas. Surg Neurol Int 2013;4(01):71

16 Claus EB, Calvocoressi L, Bondy ML, Schildkraut JM, Wiemels JL, Wrensch M. Family and personal medical history and risk of meningioma. J Neurosurg 2011;115(06):1072-1077

17 Matsuda Y, Kawamoto K, Kiya K, Kurisu K, Sugiyama K, Uozumi T. Antitumor effects of antiprogesterones on human meningioma cells in vitro and in vivo. J Neurosurg 1994;80(03): 527-534

18 Olson JJ, Beck DW, Schlechte J, Loh PM. Hormonal manipulation of meningiomas in vitro. J Neurosurg 1986;65(01):99-107

19 Grunberg SM, Weiss MH, Spitz IM, et al. Treatment of unresectable meningiomas with the antiprogesterone agent mifepristone. J Neurosurg 1991;74(06):861-866

20 Ji Y, Rankin C, Grunberg S, et al. Double-blind phase III randomized trial of the antiprogestin agent mifepristone in the treatment of unresectable meningioma: SWOG s9005. J Clin Oncol 2015;33 (34):4093-4098

21 Knight EJ, McDonald MJ. Recurrence and progression of meningioma in male-to-female transgender individuals during exogenous hormone use. Int J Transgenderism 2013;14 (01):18-23

22 Vadivelu S, Sharer L, Schulder M. Regression of multiple intracranial meningiomas after cessation of long-term progesterone agonist therapy. J Neurosurg 2010;112(05):920-924

23 Peyre M, Gaillard S, de Marcellus C, et al. Progestin-associated shift of meningioma mutational landscape. Ann Oncol 2017 (February):1-6

24 Shahin MN, Magill ST, Dalle Ore CL, et al. Fertility treatment is associated with multiple meningiomas and younger age at diagnosis. J Neurooncol 2019;143(01):137-144

25 Raj R, Korja M, Koroknay-Pál P, Niemelä M Multiple meningiomas in two male-to-female transsexual patients with hormone replacement therapy: a report of two cases and a brief literature review. Surg Neurol Int 2018;9(01):109

26 Ter Wengel PV, Martin E, Gooren L, Den Heijer M, Peerdeman SM. Meningiomas in three male-to-female transgender subjects using oestrogens/progestogens and review of the literature. Andrologia 2016;48(10):1130-1137 